\title{
Evaluation of Inter-session 3D-TRUS to 3D-TRUS Image Registration for Repeat Prostate Biopsies
}

\author{
Vaishali V. Karnik ${ }^{1,2}$, Aaron Fenster ${ }^{1,2,3}$, Jeff Bax ${ }^{1,2}$, Lori Gardi ${ }^{2}$, Igor Gyacskov ${ }^{2}$ \\ Jacques Montreuil $^{2}$, Cesare Romagnoli ${ }^{2}$, and Aaron D. Ward ${ }^{2}$ \\ ${ }^{1}$ Biomedical Engineering Graduate Program \\ ${ }^{2}$ Robarts Research Institute \\ ${ }^{3}$ Department of Medical Biophysics, The University of Western Ontario, \\ London, ON, Canada, N6A 5C1
}

\begin{abstract}
To ensure accurate targeting and repeatability, 3D TRUS-guided biopsies require registration to determine coordinate transformations to (1) incorporate pre-procedure biopsy plans and (2) compensate for inter-session prostate motion and deformation between repeat biopsy sessions. We evaluated prostate surface- and image-based 3D-to-3D TRUS registration by measuring the TRE of manually marked, corresponding, intrinsic fiducials in the whole gland and peripheral zone, and also evaluated the error anisotropy. The imagebased rigid and non-rigid methods yielded the best results with mean TREs of $2.26 \mathrm{~mm}$ and $1.96 \mathrm{~mm}$, respectively. These results compare favorably with a clinical need for an error of less than $2.5 \mathrm{~mm}$.
\end{abstract}

Keywords: surface-based registration, image-based registration, accuracy, prostate, repeat biopsy, inter-session, 3D TRUS, peripheral zone.

\section{Introduction}

Current diagnostic tests for prostate cancer $(\mathrm{PCa})$ include prostate-specific antigen (PSA) tests and digital rectal exams (DRE), but two-dimensional (2D) transrectal ultrasound (TRUS) guided biopsy $(\mathrm{Bx})$ is the clinical standard for definitive diagnosis. 2D TRUS guidance, however, is not without limitations. A 2D view restricts the anatomic information available to the physician for the accurate characterization of tumor location, which is vital for targeting and needle guidance. PCa is particularly difficult to target with 2D TRUS-guided Bx since this cancer tends to be small and multifocal. Furthermore, up to $80 \%$ of the cancer can be located in the prostate's peripheral zone (PZ) [1], an area subject to deformation due to ultrasound transducer pressure during the exam (Fig. 1). These difficulties contribute to false negative rates as high as $34 \%$ [2], and the detection rate of cancer on the necessary repeat Bx ranges between $10 \%$ and $25 \%$ [3]. In order to reduce the need for repeat Bxs, and provide better planning for required repeat Bxs, improved techniques are necessary to accurately identify tumor locations and record biopsy cores so as to avoid negative areas and target precancerous lesions. Techniques for obtaining an accurate 3D record of Bx locations and providing guidance of repeated Bxs to specific locations are of 
profound importance to the timely diagnosis of prostate cancer, and lead to high impact on patient care and quality of life.

A three-dimensional (3D) TRUS-guided prostate biopsy system has been developed in our laboratory, using a mechanically stabilized and tracked end-firing probe that provides a $3 \mathrm{D}$ view of the anatomy and records the 3D location of each Bx core (Fig. 1(c)) [4]. In order to accurately transform the 3D locations of Bx cores taken during a previous session into the coordinate space of the current session, 3D-TRUS to 3DTRUS image registration needs to be performed. The transformation given by this registration will compensate for differences in prostate position and deformation between sessions, which can be substantial due to the time between biopsy sessions, and may be due to differences in patient positioning, edema and swelling due to needle insertion, and bladder and/or rectum filling.

In this study, we evaluate the accuracy of four algorithms for 3D-TRUS to $3 D$ TRUS registration of prostate images taken during different biopsy sessions. We evaluate rigid and non-rigid variations of both surface-based and image-based registration algorithms. We determine a mean target registration error (TRE) for each algorithm and provide an analysis of the spatial anisotropy in the TRE. Furthermore, since the PZ of the prostate is of particular clinical interest, and we hypothesize that this region may undergo characteristically different (from the remainder of the gland) deformations due to its proximity to the ultrasound transducer, we perform a separate accuracy analysis on this region. Our primary success criterion is a clinically desired TRE of $2.5 \mathrm{~mm}$ or less, with a non-dominant fiducial localization error (FLE) [5].

Previous related work focuses on intra-session registration of 3D-TRUS to 3DTRUS [5, 6], and 3D-TRUS to magnetic resonance (MR) [7] prostate images, and accuracies as high as $1.50 \mathrm{~mm}$ have been reported. However, to the best of our knowledge, an evaluation of 3D-TRUS to 3D-TRUS inter-session registration, with its attendant challenges as described above, has not been previously conducted for image-guided prostate biopsies.

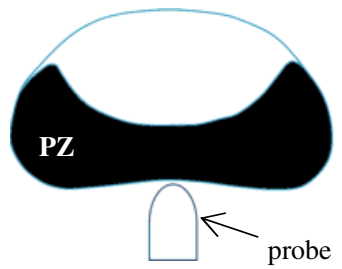

(a)

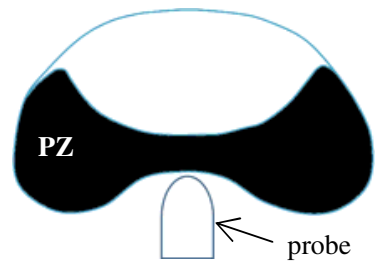

(b)

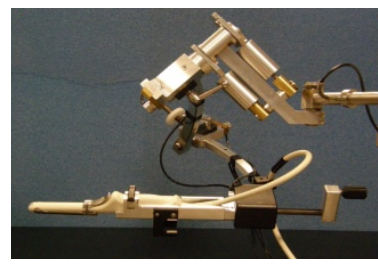

(c)

Fig. 1. Axial view of prostate with PZ highlighted in black (a) without deformation and (b) with deformation caused by transducer probe pressure. (c) Mechanically assisted 3D-TRUS biopsy system with real-time tracking and recording of the 3D position of the biopsy needle.

\section{Materials and Methods}

\subsection{Materials}

3D TRUS prostate images were obtained from 10 different patients who had undergone two prostate imaging sessions one week apart. The physician inserts the 
transducer probe into the patient's rectum and rotates it (by rolling it about the probe axis) to acquire $1802 \mathrm{D}$ images in 1 degree increments. The 2D US images from the US machine are digitized using a frame grabber and reconstructed into a 3D image. Acquisition of the 3D image requires approximately 10 seconds and the reconstruction occurs as the images are acquired, allowing the physician to view the $3 \mathrm{D}$ image as it is being acquired. The first set of scans was acquired using a HDI-3500 ultrasound machine and second was acquired using a HDI-5000. The same transducer probe (Philips C9-5) was used with both machines, but calibrated differently. From each patient, 2 images from the two different procedures were selected to be the source $\left(I_{S}\right)$ and target images $\left(I_{T}\right)$. The image dimensions were $448 \times 448 \times 350$ (voxel size of $0.19 \times 0.19 \times 0.19 \mathrm{~mm}^{3}$ ). The selection of images for this study was based on our ability to localize in 3D a set of corresponding intrinsic fiducials (small calcifications) in the image pairs for use in computing the TRE. In all 10 image pairs, we found a total of 92 fiducial pairs, of which 18 were located in the PZ. It is important to note that the fiducials in our study are used only to evaluate the registration methods, and are not used as inputs to the registration algorithms.

\subsection{Surface-Based Registration}

For surface-based registration, we first segmented the images semi-automatically using a dynamically deformable contour model [8]. We then rigidly registered the surfaces using the iterative closest point (ICP) algorithm [9], where both surfaces are represented by point clouds. In order to align the surfaces, a rigid transformation $\mathrm{T}$ is required that maps each point in the source image of patient $i$, $\left(I_{S}^{i}\right)$, to its corresponding point in the target image of patient $i,\left(I_{T}^{i}\right)$. The algorithm proceeds by (1) establishing correspondence between closest point pairs, and (2) by estimating the transformation required to align the corresponding points. Steps (1) and (2) are iterated until a convergence criterion (RMS of the sum of squares of the closest point distances) of $0.01 \mathrm{~mm}$ is met [9].

Following rigid registration, we performed a non-rigid registration using a thinplate spline (TPS) [10]. Parametric correspondence between surfaces $I_{S}^{i}$ and $I_{T}^{i}$ was established by radially projecting equal-angle rays from the centroid of the rigidly aligned surfaces, and corresponding the point pair lying on each ray. This approach to correspondence establishment is justified due to the approximately spherical shape of the prostate. A 3D TPS is defined in the 3D image space and aligns the corresponding points. The TPS is applied to $I_{S}^{i}$ in order to register it to $I_{T}^{i}$.

\subsection{Image-Based Registration}

We adopt the block matching approach proposed by Ourselin et al. for rigid registration [11]. The registration consists of the following four steps: (1) dividing the source image into rectangular block regions; (2) for each block in $I_{S}^{i}$, finding the block in a local neighborhood within $I_{T}^{i}$ having the optimal value of a chosen image-to-image metric; (3) constructing a vector field mapping each $I_{S}^{i}$ block to its best matching $I_{T}^{i}$ 
block; and (4) performing a least trimmed squares optimization to regularize the vector fields [12] in order to determine the transformation mapping $I_{S}^{i}$ to $I_{T}^{i}$. Steps (1) through (4) are iterated, with a progressively smaller block size, in order to provide a coarse-to-fine registration of the images. The chosen image-to-image metric is mutual information [13], due primarily to the effects of directionally-dependent shadowing artifacts present in 3D TRUS images. We used the implementation provided by MedINRIA (INRIA, Asclepios Research Team, 2009) that adopts a multiresolution approach. Results were achieved using a multi-resolution image pyramid where the finest and coarsest image resolutions used were tuned empirically to $32 \times 32 \times 32$ and $16 \times 16 \times 16$, respectively. The block size, $N$, is initialized to $N=\min \left(\frac{X}{8}, \frac{Y}{8}, \frac{Z}{8}\right)$, where $X, Y$ and $Z$ are the 3D image dimensions and the initial search area, $\Omega$, is equal to $2 N$ [14]. The parameters are halved when refining the scale and the process stops when the block size is $N=4$.

For image-based non-rigid registration, the moving image deformation is defined using a regular 3D grid of B-spline control points. We used a limited-memory Broyden Fletcher Goldfarb Shannon optimizer [15], and mutual information [13] as the image-to-image metric. We used the implementation provided by the 3D-SLICER software (Surgical Planning Lab, Harvard Medical School, Boston, USA), which permits the tuning of the following parameters: the number of optimization iterations (we set this parameter to 50), the number of elements along each side of the grid of Bspline control points (10), the number of histogram bins (20) and randomly-sampled voxels $(100,000)$ to use in computing the mutual information metric, and the maximum allowed deformation of the B-spline grid control points (1).

\subsection{TRE}

We measured the TRE as the overall misalignment of manually marked, corresponding, intrinsic fiducials in $I_{S}^{i}$ and $I_{T}^{i}$. The TRE is defined, for the $j$ th corresponding fiducial in patient $i$, as the Euclidean distance between the transformed fiducial $\tilde{\boldsymbol{f}}_{S}^{i j}=T\left(\boldsymbol{f}_{S}^{i j}\right)$ from $I_{S}^{i}$ and the corresponding fiducial $\boldsymbol{f}_{T}^{i j}$ in $I_{T}^{i}$, written as $T R E^{i j}=\left\|\boldsymbol{v}^{\mathrm{ij}}\right\|_{2}$, where $\boldsymbol{v}^{\mathrm{ij}}=\left(\boldsymbol{f}_{T}^{i j}-\tilde{\boldsymbol{f}}_{S}^{i j}\right)$. The mean TRE for patient $i$ is denoted as $T R E^{i}=\sqrt{\sum_{j}\left(T R E^{i j}\right)^{2} / N_{i}}$, where $N_{i}$ is the number of fiducials identified in $I_{S}^{i}$. The overall mean TRE is denoted as $T R E=\sqrt{\sum_{i} \sum_{j}\left(T R E^{i j}\right)^{2} / N}$, where $N$ is the total number of fiducial pairs used in the calculation. In this study, there were 92 fiducial pairs in total, of which 18 were within the PZ of the prostates.

\subsection{Error Anisotropy}

We performed a 3D principal component analysis (PCA) of the 3D point set $\boldsymbol{\Delta}=\left\{\left(\boldsymbol{f}_{T x}^{i j}-\tilde{\boldsymbol{f}}_{S x}^{i j}, \boldsymbol{f}_{T y}^{i j}-\tilde{\boldsymbol{f}}_{S y}^{i j}, \boldsymbol{f}_{T z}^{i j}-\tilde{\boldsymbol{f}}_{S z}^{i j}\right), \forall i, \forall j\right\}$ in order to evaluate the directional 
anisotropy of the TRE. Using MATLAB (The Mathworks Inc., Natick, MA), we computed the PCA by finding the eigenvectors $\left(\mathbf{e}_{\mathrm{i}}\right)$ and eigenvalues $\left(\lambda_{\mathrm{i}}\right)$ of the covariance matrix of $\boldsymbol{\Delta}$. This was done for both the whole gland (WG) and PZ. The $95 \%$ standard error ellipsoid volumes were defined with their semi-principal axes parallel to the eigenvectors, with lengths $a_{i}=\sqrt{\lambda_{i} \times \chi_{\alpha, v}^{2}},\left(i=1,2,3\right.$ and $\left.\chi_{\alpha, v} \approx 2.80\right)$, where $\chi_{\alpha, v}^{2}$ is the probability distribution evaluated for $95 \%$ confidence $(\alpha=0.05)$ and 3 degrees of freedom $(v=3)$ [16].

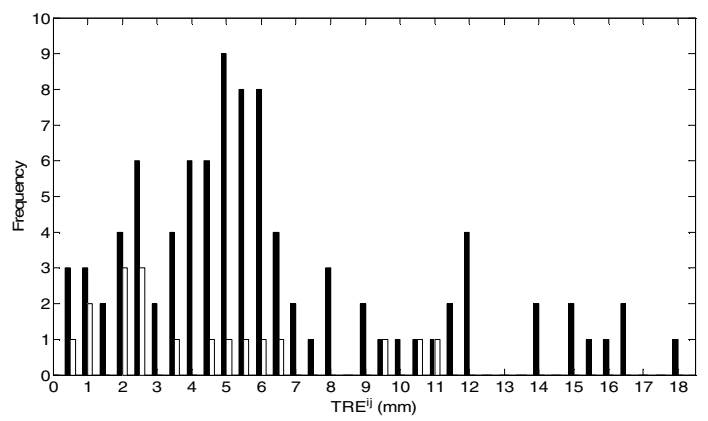

(a)

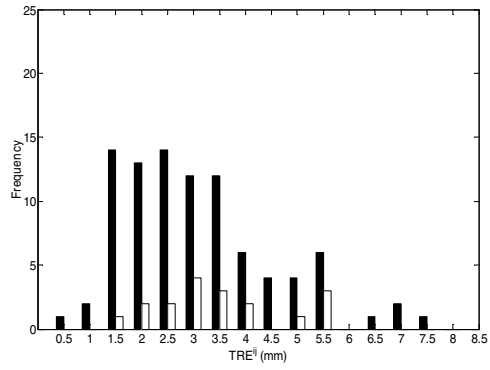

(b)

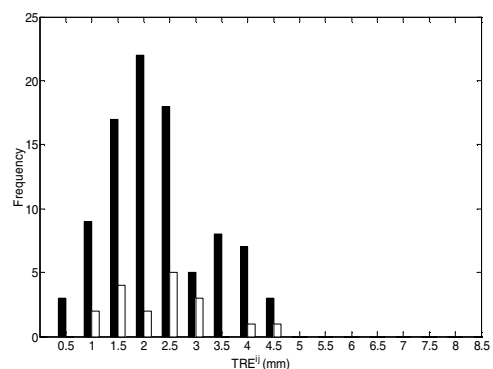

(d)

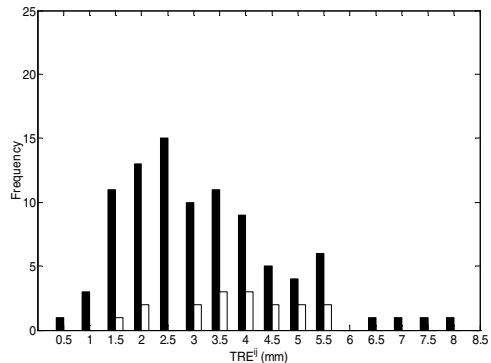

(c)

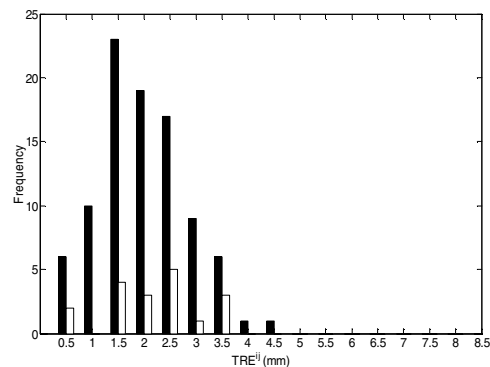

(e)

Fig. 2. WG (black) and PZ (white) frequency distributions of (a) pre-registered distances between all 92 fiducial pairs, (b) rigid surface-based TRE ${ }^{\mathrm{ij}}$, (c) non-rigid surface-based TRE ${ }^{\mathrm{ij}}$, (d) rigid image-based $\mathrm{TRE}^{\mathrm{ij}}$, (e) non-rigid image-based $\mathrm{TRE}^{\mathrm{ij}}$ 


\section{Results}

\subsection{TRE}

Our pre-registration mean misalignment was $\sim 7.45 \mathrm{~mm}$, ranging from $0.28-18.0 \mathrm{~mm}$. The frequency distributions of the WG and PZ TREs are shown in Figure 2 and the TRE results are summarized in Table 1. The two-tailed paired t-test results shown in Table 2 indicate that surface- and image-based rigid registration TREs were statistically significantly different with $\mathrm{p}=4.29 \times 10^{-6}$. The surface- and image-based non-rigid TREs were also significantly different, with $\mathrm{p}=1.60 \times 10^{-9}$.

Table 1. Whole gland (WG) and peripheral zone (PZ) mean TRE results for surface-based (SB) and image-based (IB) registration

\begin{tabular}{|c|c|c|c|c|}
\hline \multirow[t]{2}{*}{ Registration } & \multicolumn{2}{|c|}{ WG } & \multicolumn{2}{|c|}{$\mathbf{P Z}$} \\
\hline & SB & IB & SB & IB \\
\hline $\begin{array}{l}\text { Pre-registration } \\
\text { mean TRE }(\mathrm{mm})\end{array}$ & \multicolumn{2}{|c|}{$7.36 \pm 4.17$} & \multicolumn{2}{|c|}{$5.23 \pm 3.29$} \\
\hline Rigid mean TRE (mm) & $3.20 \pm 1.46$ & $2.26 \pm 0.98$ & $3.45 \pm 1.21$ & $2.30 \pm 0.90$ \\
\hline Non-rigid mean TRE(mm) & $3.29 \pm 1.50$ & $1.96 \pm 0.85$ & $3.62 \pm 1.12$ & $2.11 \pm 0.91$ \\
\hline
\end{tabular}

Table 2. t-test results for surface-based (SB), image-based (IB), rigid (R), and non-rigid (NR) registration

\begin{tabular}{ccccc}
\hline & SB vs. IB & SB vs. IB & SB & IB \\
\cline { 2 - 5 } & R & NR & R vs. NR & R vs. NR \\
\hline WG p-value & $4.29 \times 10^{-6}$ & $1.6 \times 10^{-9}$ & 0.05 & $1.38 \times 10^{-7}$ \\
PZ p-value & $1.88 \times 10^{-4}$ & $6.21 \times 10^{-6}$ & 0.12 & 0.07 \\
\hline
\end{tabular}

\subsection{Error Anisotropy}

Figure 3 shows the 95\% confidence ellipsoids for the PCA performed on the directional components of the WG and PZ TREs for the non-rigid registration methods. The WG pre-registered ellipsoid volume $(\mathrm{EV})$ is $4.46 \mathrm{~cm}^{3}$ while the post-registration EVs range from $0.12 \mathrm{~cm}^{3}$ to $0.41 \mathrm{~cm}^{3}$. The EVs for surface-based rigid and non-rigid TRE were greater than the image-based EVs by an average of $0.27 \mathrm{~cm}^{3}$.

The PZ pre-registered EV is $1.58 \mathrm{~cm}^{3}$ while the post-registration volumes range from $0.14 \mathrm{~cm}^{3}$ to $0.37 \mathrm{~cm}^{3}$. The EVs for surface-based rigid and non-rigid TRE were greater than the EVs for image-based TRE by an average of $0.22 \mathrm{~cm}^{3}$. Table 3 shows the ratios of the eigenvalues for each registration approach as well as for the unregistered case, to illustrate any anisotropy in the error. 


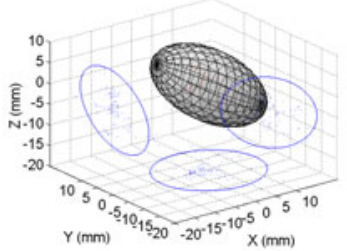

(a)

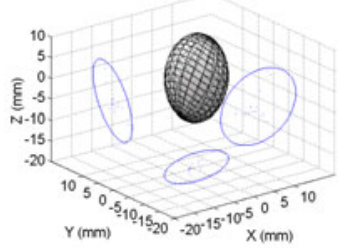

(d)

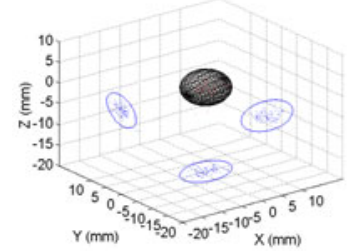

(b)

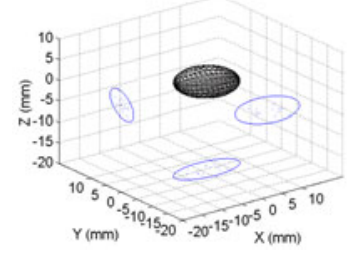

(e)

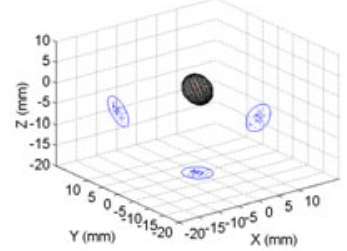

(c)

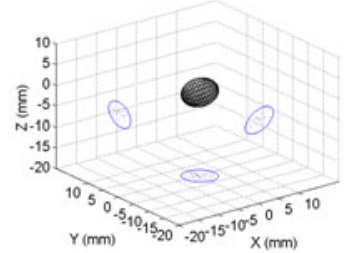

(f)

Fig. 3. Error ellipsoids for WG and PZ: (a) WG pre-registered data, (b) WG surface-based nonrigid registration, (c) WG image-based non-rigid registration, (d) PZ pre-registered data, (e) PZ surface-based non-rigid registration, and (f) PZ image-based non-rigid registration

Table 3. Ratios of $95 \%$ TRE confidence ellipsoid eigenvalues

\begin{tabular}{lcccccc}
\hline & \multicolumn{3}{c}{ WG } & \multicolumn{3}{c}{ PZ } \\
\cline { 2 - 7 } Registration Method & $\lambda_{1} / \lambda_{2}$ & $\lambda_{2} / \lambda_{3}$ & $\lambda_{1} / \lambda_{3}$ & $\lambda_{1} / \lambda_{2}$ & $\lambda_{2} / \lambda_{3}$ & $\lambda_{1} / \lambda_{3}$ \\
\hline Unregistered & 1.16 & 1.37 & 1.43 & 1.24 & 1.72 & 1.95 \\
Surface-based rigid & 1.51 & 1.36 & 1.42 & 2.08 & 1.6 & 1.6 \\
Surface-based non-rigid & 1.76 & 1.86 & 2.02 & 2.58 & 2.75 & 3.13 \\
Image-based rigid & 1.16 & 1.37 & 1.43 & 1.24 & 1.72 & 1.95 \\
Image-based non-rigid & 1.51 & 1.36 & 1.42 & 2.08 & 1.6 & 1.6 \\
\hline
\end{tabular}

\section{Discussion}

Using 3D image registration to correct for prostate motion and deformation that occurs between Bx procedures is vital to accurate Bx planning and needle guidance. The desired TRE is $2.5 \mathrm{~mm}$ since the smallest tumor considered clinically significant has a radius of $\sim 5 \mathrm{~mm}$ (for a $0.5 \mathrm{~cm}^{3}$ spherical tumor) [17]. Since the TRE (RMSE) provides an estimate of the standard deviation of the normal distribution of biopsy targets given by a registration algorithm, a TRE of $2.5 \mathrm{~mm}$ gives a confidence interval with $95.4 \%$ of the registered targets within the clinically significant $5 \mathrm{~mm}$ radius.

The non-rigid image-based method yielded the lowest WG and PZ TRE values of $1.96 \mathrm{~mm}$ and $2.11 \mathrm{~mm}$, respectively. Figures 2(d) and (e) show that the image-based method outperformed the surface-based method by illustrating the right-skewed distribution of the TRE values (for both $\mathrm{WG}$ and PZ), with $\sim 65 \%$ of the values below the respective means. In a previous evaluation of image registration methods in the intrasession context, the non-rigid image-based TRE for images taken 6 minutes apart was 
$1.50 \mathrm{~mm}$ [5]. This shows that after a time gap of 1 week, the same image-based registration algorithm, tuned as in [5], is able to achieve comparable results.

The t-test results in Table 2 reveal statistically significant differences in all cases except when comparing surface-based rigid and non-rigid registration values. We speculate that this could be due to the variability introduced by segmentation $( \pm 1.31$ $\mathrm{mm})$ [5]. The FLE [18] was $0.21 \mathrm{~mm}$ [5], and thus was not dominant.

Figure 3 shows the anisotropy in the TRE where both WG and PZ pre-registered fiducial misalignments are predominantly in the $\mathrm{z}$-direction, which is along the transducer probe axis where we might expect misalignments due to probe pressure. The surface-based errors are predominantly anisotropic in $\mathrm{x}$ and $\mathrm{y}$, which may be the result of inconsistent segmentation due to poor image contrast at the prostate boundaries parallel to the ultrasound beam. Table 3 indicates increased error anisotropy in the PZ, in comparison to the WG. This may be due to deformation that characteristically pushes the central region of the PZ upward in response to probe pressure, while the lateral "lobes" of the PZ bulge downward and laterally. The post-registration 3D EVs are dramatically reduced (by a factor of 10-37 for WG, and 4.27-11.29 for PZ) as compared with the pre-registered EVs.

Our results demonstrate that in the inter-session scenario with 1 week between sessions, the tested surface-based and image-based 3D TRUS image registration techniques yield clinically sufficient accuracy, with image-based registration giving the lowest error. Furthermore, due to the segmentation step required for surface-based registration, the two methods are comparable in execution time on a contemporary single-core workstation (90-105 s). The image-based method is preferred since it is more accurate, does not require segmentation and can potentially be automated.

\section{References}

1. Prostate Cancer Treatment Guide (2008-2010), http://www.prostate-cancer.com

2. Fink, K., et al.: Evaluation of transition zone and lateral sextant biopsies for prostate cancer detection after initial sextant biopsy. Urology 61, 748-753 (2003)

3. Kronz, D., et al.: Predicting cancer following a diagnosis of high-grade prostatic intraepithelial neoplasia on needle biopsy: Data on men with more than one follow-up biopsy. The American Journal of Surgical Pathology 25(8), 1079-1085 (2001)

4. Bax, J., et al.: Mechanically assisted 3D ultrasound guided prostate biopsy system. Medical Physics 35(12), 5397-5410 (2008)

5. Karnik, V.V., et al.: Assessment of image registration accuracy in three-dimensional transrectal ultrasound guided prostate biopsy. Medical Physics 37(2), 802-813 (2010)

6. Baumann, M., et al.: Prostate biopsy assistance system with gland deformation estimation for enhanced precision. In: Yang, G.-Z., Hawkes, D., Rueckert, D., Noble, A., Taylor, C. (eds.) MICCAI 2009. LNCS, vol. 5761, pp. 67-74. Springer, Heidelberg (2009)

7. $\mathrm{Hu}, \mathrm{Y}$., et al.: MR to ultrasound image registration for guiding prostate biopsy and interventions. In: Yang, G.-Z., Hawkes, D., Rueckert, D., Noble, A., Taylor, C. (eds.) MICCAI 2009. LNCS, vol. 5761, pp. 787-794. Springer, Heidelberg (2009)

8. Wang, Y., et al.: Semiautomatic three-dimensional segmentation of the prostate using twodimensional ultrasound images. Medical Physics 30(5), 887-897 (2003) 
9. Besl, P., McKay, N.: A method for registration of 3-D shapes. IEEE Trans. on Pattern Analysis and Machine Intelligence 14, 239-256 (1992)

10. Bookstein, F., et al.: Principal warps: Thin-plate splines and the decomposition of deformations. IEEE Trans. on Pattern Analysis and Machine Intelligence 11(6), 567-585 (1989)

11. Ourselin, S., et al.: Block matching: A general framework to improve robustness of rigid registration of medical images. In: Niessen, W.J., Viergever, M.A. (eds.) MICCAI 2001. LNCS, vol. 2208, pp. 557-566. Springer, Heidelberg (2001)

12. Rousseuw, P.J., Leroy, A.M.: Robust regression and outlier detection, 1st edn. Wiley Series in Probability and Mathematical Statistics (1987)

13. Wells III, M., et al.: Multi-modal volume registration by maximization of mutual information. Medical Image Analysis 1(1), 35-51 (1996)

14. Ourselin, S., et al.: Reconstructing a 3D structure from serial histological sections. Image and Vision Computing 19, 25-31 (2000)

15. Zhu, C., et al.: L-BFGS-B: Algorithm 778: L-BFGS-B, FORTRAN routines for large scale bound constrained optimization. ACM TMS 23(4), 550-560 (1997)

16. Irwin, M., et al.: Registered 3-D ultrasound and digital stereotactic mammography for breast biopsy guidance. IEEE TMI 27(3), 391-398 (2008)

17. Epstein, J.I., et al.: Utility of saturation biopsy to predict insignificant cancer at radical prostatectomy. Urology 66(2), 356-360 (2005)

18. Fitzpatrick, J.M., et al.: Predicting error in rigid-body point-based registration. IEEE TMI 17(5), 694-702 (1998) 